\title{
Novel Relay Selection Strategies for Cooperative NOMA
}

DOI:

10.1109/TVT.2017.2752264

\section{Document Version}

Accepted author manuscript

Link to publication record in Manchester Research Explorer

\section{Citation for published version (APA):}

Yang, Z., Ding, Z., Wu, Y., \& Fan, P. (2017). Novel Relay Selection Strategies for Cooperative NOMA. IEEE Transactions on Vehicular Technology. https://doi.org/10.1109/TVT.2017.2752264

\section{Published in:}

IEEE Transactions on Vehicular Technology

\section{Citing this paper}

Please note that where the full-text provided on Manchester Research Explorer is the Author Accepted Manuscript or Proof version this may differ from the final Published version. If citing, it is advised that you check and use the publisher's definitive version.

\section{General rights}

Copyright and moral rights for the publications made accessible in the Research Explorer are retained by the authors and/or other copyright owners and it is a condition of accessing publications that users recognise and abide by the legal requirements associated with these rights.

\section{Takedown policy}

If you believe that this document breaches copyright please refer to the University of Manchester's Takedown Procedures [http://man.ac.uk/04Y6Bo] or contact uml.scholarlycommunications@manchester.ac.uk providing relevant details, so we can investigate your claim.

\section{OPEN ACCESS}




\title{
Novel Relay Selection Strategies for Cooperative NOMA
}

\author{
Zheng Yang, Zhiguo Ding, Senior Member, IEEE, Yi Wu, Member, IEEE, and Pingzhi Fan, Fellow, IEEE
}

\begin{abstract}
In this paper, we consider non-orthogonal multiple access (NOMA) relaying networks, where one base station communicates with two mobile users with the aid of multiple relays. We propose a two-stage relay selection strategy for NOMA networks with decode-and-forward (DF) and amplifyand-forward (AF) relaying protocols with different quality of service requirements at the users, respectively. Then, the outage probabilities of the NOMA two-stage DF and AF schemes are obtained in closed-form expressions, and the diversity order is determined based on their asymptotic expressions at high signalto-noise ratio. Both of the developed analytical results and carried out computer simulations show that NOMA two-stage DF (AF) relaying is superior to existing relay selection strategies developed for cooperative NOMA and orthogonal multiple access networks.
\end{abstract}

Index Terms-Cooperative non-orthogonal multiple access, amplify-and-forward, decode-and-forward, outage probability.

\section{INTRODUCTION}

Because of its superior spectral efficiency, non-orthogonal multiple access (NOMA) has lately attracted much attention, and NOMA has been recognized as a promising multiple access candidate scheme for the next generation mobile communication networks [1] and [2]. Furthermore, downlink NOMA has recently been adopted by 3 GPP long term evolution (LTE) [3], namely, multiple user superposition transmission (MUST).

The basic concept of NOMA is to exploit the power domain and channel quality difference among users to achieve multiple access, where users can be served at the same time/frequency/code [2]. Meanwhile, successive interference cancellation (SIC) is implemented at the users to decode the signals for those users whose channel conditions are poor, then remove these decoded messages, and lastly detect their own information [4].

The outage probability and average sum rate for downlink NOMA with partial channel information have been studied in [5] and [6], where users are randomly placed in a cell.

Copyright (c) 2015 IEEE. Personal use of this material is permitted. However, permission to use this material for any other purposes must be obtained from the IEEE by sending a request to pubs-permissions@ieee.org.

Z. Yang and Y. Wu are with the Fujian Provincial Engineering Technology Research Center of Photoelectric Sensing Application, Fujian Normal University, Fuzhou 350007, P. R. China. (email: zyfjnu@163.com, wuyi@fjnu.edu.cn). (Corresponding author: Yi Wu)

Z. Ding is with the School of Computing and Communications, Lancaster University, LA1 4YW, UK. (e-mail: z.ding@lancaster.ac.uk).

P. Fan is with the Institute of Mobile Communications, Southwest Jiaotong University, Chengdu 610031, P. R. China. (email: p.fan@ieee.org).

The work of Z. Yang and Y. Wu were supported by National Natural Science Foundation of China under Grant 61701118 and National Natural Science Foundation of China under Grant 61571128.

The work of Z. Ding was supported by the UK EPSRC under grant number EP/L025272/1 and by H2020-MSCA-RISE-2015 under grant number 690750.

The work of P. Fan was supported by the National Sci \& Tech Major Project under Grant No. 2016ZX03001018, and the 111 Project under Grant No. 111-2-14
These analytical results draw a conclusion that NOMA outperforms traditional orthogonal multiple access (OMA). Another conclusion is that in contrast to OMA, the strong user in NOMA can significantly strengthen its performance, but the performance gain for the weak user is relatively small. A natural idea is that combining relay transmission with NOMA can enhance the weak user's performance. Cooperative NOMA was firstly proposed in [7], where the user having strong channel conditions can act as a relay to help the user suffering from weak channel conditions because SIC is implemented at the strong user.

Recall that there are two well known relay protocols, e.g., decode-and-forward (DF) and amplify-and-forward (AF), which have been commonly used in cooperative communication networks [8] and [9]. In [10], the authors have studied the performance of the scenario in which one source communicates with multiple receivers via an AF relay by using NOMA under Nakagami-m fading, while one source communicates with two users through multiple NOMA AF relaying under Rayleigh fading is considered in [11]. It can be concluded that NOMA relaying networks can yield better performance than OMA relaying systems when users' power allocation coefficients and target date rates are well chosen [10] [11]. Furthermore, the NOMA AF relaying networks in [10] have been extended to a case with one source and multiple receivers each equipped with multiple antennas [12]. A NOMA DF relaying model has been proposed in [13], in order to enhance cell edge user performance. Following the cooperative NOMA model in [7], the concept of simultaneous wireless information and power transfer has been applied at the strong user to aid the base station for serving the weak user [14]. Recently, a two-stage max-min relay selection scheme was proposed for cooperative NOMA in [15], i.e., the first stage is to find a subset of the relays which can successfully decode the message intended to the user with a low rate requirement, then the max-min scheme is applied to select a relay from the subset in order to serve the user which requires a high rate. The outage performance for the two-stage max-min relay selection approach is superior to that of the conventional max-min strategy. However, the qualified selected relays in the first stage cannot always correctly decode the message intended to the user with a high data rate requirement, which will lead to a loss performance gain. Furthermore, fixed power allocation was considered in [15], which leads to a situation that the outage probability always occurs if one chooses a wrong choice for the power allocation coefficients.

In this paper, we consider a cooperative NOMA system with one transmitter communicates with two receivers via multiple relays. The major contributions of this paper are organized as follows.

- A new two-stage relay selection scheme is proposed in 
cooperative NOMA networks, where one user's quality of service (QoS) can be strictly satisfied in the first stage, while maximizing its partner's rate in the second stage. We then combine DF and AF relaying with this two-stage approach, respectively. Unlike the two-stage max-min scheme in [15], the users' power allocation coefficients are related to the relay-destination channels in two-stage DF relaying, and they are functions of the source-relay and relay-destination channels in two-stage AF relaying.

- According to the proposed power allocation factors, we obtain the exact expressions for the outage probabilities for the users in two-stage DF and AF relaying NOMA, respectively, as well as their asymptotic expressions at high signal-to-noise ratio (SNR) in order to analyze the diversity gain. The analytical expressions demonstrate that the outage performance for NOMA two-stage DF (AF) relaying is better than those in conventional OMA $\mathrm{DF}$ (AF) relaying, although they realize the same diversity order.

- Recall that the coverage probability in the NOMA twostage max-min strategy will be always one without choosing the users' target rates and power allocation factors in a correct way [15]. This new NOMA two-stage AF relaying can avoid such a disadvantage and is applicable with more flexible parameter selection. Furthermore, this new two-stage AF relaying can achieve the best outage probability among those existing relay selection schemes for cooperative NOMA.

This paper is organized as follows. In Section II, the system model and two relay selection schemes are introduced. Section III and Section IV propose the two-stage DF and AF relay selection scheme for cooperative NOMA, respectively, and investigate the outage probability is achieved by the proposed schemes. Numerical results are presented in Section $\mathrm{V}$ to demonstrate the outage performance of the proposed schemes. Finally, Section VI concludes the paper.

\section{System Model}

Consider a cooperative NOMA system which contains one source, $M$ relays and two users. Assume that all nodes are equipped with a single antenna, and there is no direct link between the source and the users. The source communicates with the users through a chosen DF or AF relay from the $M$ available relays. Furthermore, assume that all links undergo quasi-static Rayleigh fading. Recall that the users are ordered by the quality of their channels from the relays to users in cooperative NOMA [10] and [12]. Unlike these existing works, we assume that channel conditions between the relays and users are statistically the same, i.e., the relay-user channel fading gains are assumed to be independent and identically distributed, and the users are not ordered based on their channel conditions. Instead, the users are ordered by their different QoS requirements [15] and [16], e.g., the users are distinguished not by their channel conditions, but their QoS requirements. Without loss of generality, assume that the user $U_{1}$ has a low target rate but requires to be served quickly, such as an Internet of Things sensor or a healthcare device. On the other hand, the user $U_{2}$ would like to download a movie with high resolution. The two-phase signal transmission schemes for DF and AF relaying are presented in the following two subsections.

\section{A. DF Relaying}

In the first time slot, the source transmits a superposition signal, $x_{s}=\sum_{i=1}^{2} \sqrt{\gamma_{i}} x_{i}$, to $M$ relays, where $x_{i}$ is the signal for $U_{i}, \gamma_{i}$ denotes the power allocation factor for message $x_{i}$ with $\sum_{i=1}^{2} \gamma_{i}=1$ and $\gamma_{i}>0$. Therefore, the received signal at relay $n, 1 \leq n \leq M$, is given by

$$
y_{n}^{r}=\sqrt{P} \sum_{i=1}^{2} \sqrt{\gamma_{i}} x_{i} g_{n}+\omega_{n}^{r},
$$

where $P$ and $\omega_{n}^{r}$ denote the transmission power at the source and the additive white Gaussian noise (AWGN) with zero mean and variance $\sigma^{2}$, and $g_{n}$ is the channel coefficient from the source to relay $n$.

Due to the fact that the QoS requirements of $U_{1}$ are much less demanding than those in $U_{2}$, we assume that the decoding order is always from $U_{1}$ to $U_{2}$. Therefore, the achievable rates for relay $n$ to decode the signals $x_{1}$ and $x_{2}$ are given by [15]

$$
R_{1}^{n}=\frac{1}{2} \log _{2}\left(1+\frac{\gamma_{1} \rho\left|g_{n}\right|^{2}}{1+\gamma_{2} \rho\left|g_{n}\right|^{2}}\right),
$$

and

$$
R_{2}^{n}=\frac{1}{2} \log _{2}\left(1+\gamma_{2} \rho\left|g_{n}\right|^{2}\right),
$$

respectively, where $\rho=\frac{P}{\sigma^{2}}$ denotes the transmission SNR. Note that the $R_{2}^{n}$ in (3) is achievable under the condition that $R_{1}^{n} \geq R_{1}$, i.e., the SIC is successfully carried out at relay $n$ to remove the signal $x_{1}$, where $R_{1}$ denotes the target data rate of $U_{1}$.

If relay $n$ can successfully decode both the signals $x_{1}$ and $x_{2}$ in the first phase, the relay $n$ can use NOMA to broadcast a superimposed mixture to the users in the second phase. Assume the superimposed mixture is $x_{n}^{r}=\sum_{i=1}^{2} \sqrt{\alpha_{i}} x_{i}$, where $\alpha_{i}$ is the power allocation coefficient for $U_{i}$ with $\alpha_{1}+\alpha_{2}=1$ and $\alpha_{i}>0$. Therefore, the observation signals at $U_{k}, k=1,2$ can be written as follows:

$$
y_{k}^{d}=\sqrt{P} h_{n k} \sum_{i=1}^{2} \sqrt{\alpha_{i}} x_{i}+\omega_{k}^{d},
$$

where $h_{n k}$ is the channel coefficient from relay $n$ to $U_{k}, P$ denotes the transmission power at relay $n$, and $\omega_{k}^{d}$ denotes the AWGN at $U_{k}$, i.e., $\omega_{k}^{d} \sim \mathcal{C N}\left(0, \sigma^{2}\right)$.

According to (4), the instantaneous rate for $U_{1}$ to detect its signal $x_{1}$ is given by

$$
R_{1}^{d}=\frac{1}{2} \log _{2}\left(1+\frac{\alpha_{1} \rho\left|h_{n 1}\right|^{2}}{1+\alpha_{2} \rho\left|h_{n 1}\right|^{2}}\right) .
$$

The SIC is carried out at $U_{2}$ to remove the signal for $U_{1}$, therefore the instantaneous rate for $U_{2}$ to detect the signal $x_{1}$ is given by

$$
R_{1 \rightarrow 2}^{d}=\frac{1}{2} \log _{2}\left(1+\frac{\alpha_{1} \rho\left|h_{n 2}\right|^{2}}{1+\alpha_{2} \rho\left|h_{n 2}\right|^{2}}\right) .
$$


Under the condition that $R_{1 \rightarrow 2}^{d} \geq R_{1}$, the observation at $U_{2}$ in (4) can remove the signal $x_{1}$ before detecting its own message. Therefore, the achievable rate for $U_{2}$ to decode the message $x_{2}$ is given by

$$
R_{2}^{d}=\frac{1}{2} \log _{2}\left(1+\alpha_{2} \rho\left|h_{n 2}\right|^{2}\right) .
$$

Note that the decoding order of most existing works about NOMA, such as [5] and [10], relies on using the users' channel conditions. The shortcoming is that all the relays need to know the relay-user channel information, which will significantly increase the system overhead, especially in cooperative NOMA networks with a large number of users or relays. However, in this paper, the user ordering is based on the users' QoS requirements, which means NOMA can be implemented even if users have statistically the same channel conditions and the relays do not need to know the CSI for the channels between the relays and the users.

\section{B. AF Relaying}

Recall that the signal received by relay $n$ in the first time slot is $y_{n}^{r}$ in (1). In the second time slot, relay $n$ amplifies the received signal $y_{n}^{r}$ by a coefficient $\beta$, and then broadcasts it to the users in AF relaying. Therefore, the received signal at $U_{k}$ can be expressed as follows:

$$
\begin{array}{r}
y_{k}^{d}=\beta \sqrt{P} g_{n}\left(\sqrt{\gamma_{1} P} x_{1}+\sqrt{\gamma_{2} P} x_{2}\right) h_{n k} \\
+\beta \sqrt{P} h_{n k} \omega_{n}^{r}+\omega_{k}^{d},
\end{array}
$$

where the amplify factor is defined as follows:

$$
\beta=\frac{1}{\sqrt{P\left|g_{n}\right|^{2}+\sigma^{2}}} .
$$

Similar to cooperative NOMA with DF relaying, $U_{1}$ in NOMA AF relaying will firstly decode its signal $x_{1}$. Therefore, the received signal-to-interference-plus-noise ratio (SINR) for $U_{1}$ to decode message $x_{1}$ based on (8) and (9), is given by

$$
\mathrm{SINR}_{1}=\frac{\gamma_{1} \rho^{2}\left|g_{n}\right|^{2}\left|h_{n 1}\right|^{2}}{\rho^{2}\left|g_{n}\right|^{2}\left|h_{n 1}\right|^{2} \gamma_{2}+\rho\left|g_{n}\right|^{2}+\rho\left|h_{n 1}\right|^{2}+1} .
$$

Furthermore, the received SINR for $U_{2}$ to decode message $x_{1}$ is given as follows:

$$
\operatorname{SINR}_{1 \rightarrow 2}=\frac{\gamma_{1} \rho^{2}\left|g_{n}\right|^{2}\left|h_{n 2}\right|^{2}}{\rho^{2}\left|g_{n}\right|^{2}\left|h_{n 2}\right|^{2} \gamma_{2}+\rho\left|g_{n}\right|^{2}+\rho\left|h_{n 2}\right|^{2}+1} .
$$

Note that signal $x_{1}$ can be correctly decoded by $U_{2}$, if $\frac{1}{2} \log _{2}\left(1+\mathrm{SINR}_{1 \rightarrow 2}\right) \geq R_{1}$, i.e., $x_{1}$ can be removed from $y_{2}^{d}$. Therefore, $U_{2}$ detects its own message with the following SINR:

$$
\operatorname{SINR}_{2}=\frac{\gamma_{2} \rho^{2}\left|g_{n}\right|^{2}\left|h_{n 2}\right|^{2}}{\rho\left|g_{n}\right|^{2}+\rho\left|h_{n 2}\right|^{2}+1} .
$$

By using the proposed two-stage DF and AF relay selection schemes in Section II A and B, the outage performance of cooperative NOMA networks with the proposed schemes will be studied in the following two sections. The outage probability is considered as the performance evaluation criterion due to the following reason.
The outage probability of cooperative DF relaying networks is defined as the probability that neither the instantaneous rates at the relays nor the instantaneous rates at the destinations are larger than or equal to the users' date target rates. For cooperative AF relaying networks, the outage probability is directly determined by the event that the instantaneous rates at the destinations are less than the users' targeted date rates. Note that in delay-sensitive networks, the throughput mainly depends on the outage probability, since a fixed rate is transmitted by the transmitter. Therefore, the outage probability is a fundamental performance metric of delay-sensitive cooperative NOMA networks.

On the other hand, given the use of optimal channel coding with an infinite coding length, the outage probability provides a tight bound on the error probability of decoding [17]. While the bit error rate (BER) is another commonly used criterion, it is worth pointing out that evaluating the BER requires to specify the used channel coding and modulation.

\section{Cooperative NOMA with Two-Stage DF Relay SELECTION}

In this section, we firstly propose a two-stage DF relay selection scheme, then investigate its outage performance.

For a given relay $n$, only if the instantaneous rates for $U_{1}$ and $U_{2}$ are larger or equal to their target data rates $R_{1}$ and $R_{2}$, respectively, the relay can successfully decode the messages $x_{1}$ and $x_{2}$, i.e.,

$$
\left\{\begin{array}{l}
\frac{1}{2} \log _{2}\left(1+\frac{\gamma_{1}\left|g_{n}\right|^{2}}{\frac{1}{\rho}+\gamma_{2}\left|g_{n}\right|^{2}}\right) \geq R_{1}, \\
\frac{1}{2} \log _{2}\left(1+\gamma_{2} \rho\left|g_{n}\right|^{2}\right) \geq R_{2},
\end{array}\right.
$$

Based on the rate requirements of the users, we can choose different values for $R_{1}$ and $R_{2}$, and we will demonstrate how the $R_{1}$ and $R_{2}$ affect the outage performance in the numerical result section.

Based on (13), we can find that ${ }^{1}$

$$
\left|g_{n}\right|^{2}>\frac{\eta}{\rho}
$$

where $\eta=\max \left\{\frac{\varepsilon_{1}}{\gamma_{1}-\gamma_{2} \varepsilon_{1}}, \frac{\varepsilon_{2}}{\gamma_{2}}\right\}, \varepsilon_{1}=2^{2 R_{1}}-1, \varepsilon_{2}=2^{2 R_{2}}-1$. Note that if we use (14) as the constraint, which can ensure that the relay $n$ can correctly decode both $x_{1}$ and $x_{2}$.

Recall that $U_{1}$ has the priority to be served, i.e., the target rate requirements of $U_{1}$ need to be met as follows:

$$
\frac{1}{2} \log _{2}\left(1+\frac{\alpha_{1} \rho\left|h_{n 1}\right|^{2}}{1+\alpha_{2} \rho\left|h_{n 1}\right|^{2}}\right) \geq R_{1} .
$$

Based on (15), we can find that the maximal power allocation factor $\alpha_{2}$ can be expressed as follows:

$$
\alpha_{2}=\max \left\{0, \frac{\rho\left|h_{n 1}\right|^{2}-\varepsilon_{1}}{\rho\left|h_{n 1}\right|^{2}\left(1+\varepsilon_{1}\right)}\right\} .
$$

Note that if $\left|h_{n 1}\right|^{2}>\frac{\varepsilon_{1}}{\rho}$, the condition in (15) is always possible. That is to say, $U_{1}$ can always successfully detect its own message under the condition $\left|h_{n 1}\right|^{2}>\frac{\varepsilon_{1}}{\rho}$.

${ }^{1}$ Please note that the probability for the event that this constraint cannot be satisfied will be taken into consideration for the outage probability calculation. 
According to (14)-(16), a set of active relays which can satisfy the QoS requirements of $U_{1}$ can be defined as follows:

$$
S_{n}=\left\{n:\left|g_{n}\right|^{2}>\frac{\eta}{\rho},\left|h_{n 1}\right|^{2} \geq \frac{\varepsilon_{1}}{\rho}\right\} .
$$

In $S_{n}$, only the best relay is selected to serve $U_{2}$, which can be defined as follows:

$$
n^{*}=\arg \max _{n \in S_{n}}\left\{\left|h_{n 2}\right|^{2}\right\} .
$$

Furthermore, in order to ensure $U_{2}$ can achieve a high data rate, we impose a constraint that the SIC can be successfully carried out at $U_{2}$, i.e., $\frac{1}{2} \log _{2}\left(1+\frac{\alpha_{1} \rho\left|h_{\left.n_{2}\right|_{2}}\right|^{2}}{1+\alpha_{2} \rho\left|h_{n_{2}}\right|^{2}}\right) \geq R_{1}$. Again, please note that the impact of this constraint will be taken into consideration for the outage probability. Then the maximal power allocation factor $\alpha_{2}$ can be written as follows:

$$
\alpha_{2}=\max \left\{0, \frac{\rho\left|h_{n^{*} 2}\right|^{2}-\varepsilon_{1}}{\rho\left|h_{n^{*} 2}\right|^{2}\left(1+\varepsilon_{1}\right)}\right\} \text {. }
$$

Based on (16)-(19), the power allocation factor $\alpha_{2}$ for $U_{2}$ can be rewritten as follows:

$\alpha_{2}=\min \left\{\frac{\rho\left|h_{n^{*} 1}\right|^{2}-\varepsilon_{1}}{\rho\left|h_{n^{*} 1}\right|^{2}\left(1+\varepsilon_{1}\right)}, \max \left\{0, \frac{\rho\left|h_{n^{*} 2}\right|^{2}-\varepsilon_{1}}{\rho\left|h_{n^{*} 2}\right|^{2}\left(1+\varepsilon_{1}\right)}\right\}\right\}$.

It can be observed from (20) that the power allocation coefficient $\alpha_{2}$ depends on $\varepsilon_{1}$ and the channel fading gains from the best relay to the two users. Furthermore, note that if $\left|h_{n^{*} 2}\right|^{2}<\frac{\varepsilon_{1}}{\rho}$, the power allocation factor becomes zero, $\alpha_{2}=0$, since the relay allocates all the power to serve $U_{1}$, especially when the target rate of the $U_{1}$ is high or the channel condition between the best relay and $U_{2}$ is poor.

According to the above discussion of the two-stage DF relay selection scheme, we provide an exact expression for the outage probability and its diversity gain achieved by this scheme as follows.

Theorem 1: The outage probability for $U_{2}$ in cooperative NOMA with the proposed two-stage DF relaying scheme is given by

$$
\begin{aligned}
\mathrm{P}_{2}= & e^{-\frac{a_{1}}{\rho}} \sum_{k=0}^{M}\left(\begin{array}{c}
M \\
k
\end{array}\right) e^{-\frac{a k}{\rho}}\left(1-e^{-\frac{a}{\rho}}\right)^{M-k}\left(1-e^{-\frac{a_{2}}{\rho}}\right)^{k} \\
& +\frac{a_{1}}{\rho} \sum_{k=0}^{M}\left(\begin{array}{c}
M \\
k
\end{array}\right) e^{-\frac{a k}{\rho}}\left(1-e^{-\frac{a}{\rho}}\right)^{M-k} q(k),
\end{aligned}
$$

where $a_{1}=\varepsilon_{2}\left(\varepsilon_{1}+1\right), a_{2}=a_{1}+\varepsilon_{1}, a=\eta+\varepsilon_{1}, q(k)=$ $\int_{0}^{1} e^{-\frac{a_{1} t}{\rho}}\left(1-e^{-\frac{1}{\rho}\left(\frac{\varepsilon_{1}}{t}+a_{1}\right)}\right)^{k} d t$. The diversity gain of the $U_{2}$ is given by

$$
d=-\lim _{\rho \rightarrow \infty} \frac{\mathrm{P}_{2}}{\rho}=M .
$$

Proof: See Appendix A.

One can observe from Theorem 1 that the diversity gain of cooperative NOMA with the two-stage DF relaying scheme is proportional to the number of relays, which is the same as traditional OMA with max-min and DF relaying selection. Note that the numerical results in section $\mathrm{V}$ show that compared to OMA with DF relaying, the NOMA two-stage DF scheme can realize better outage behaviour. The main reason is that the users can simultaneously share the same time/frequency/code orthogonal resource, which leads to the improvement of the spectral efficiency.

\section{Cooperative NOMA with Two-Stage AF Relay SELECTION}

In this section, we focus on how to combine AF protocol with the two-stage strategy, and apply two-stage AF relaying into cooperative NOMA. We then study the outage probability for such NOMA with two-stage AF relaying.

Since $U_{1}$ needs to be served quickly with low QoS requirements, which means $\mathrm{SINR}_{1} \geq \varepsilon_{1}$ should be hold, if possible. On the other hand, recall that SIC is carried out at $U_{2}$ to remove the signal from $U_{1}$, but the channel fading gains between the relay and the users, i.e., $h_{n 1}$ and $h_{n 2}$, are not ordered. In order to ensure $U_{2}$ can achieve a high date rate, we impose the following constraint $\operatorname{SINR}_{1 \rightarrow 2} \geq \varepsilon_{1}$, which means SIC can be successfully implemented by $U_{2}$ to remove the signal intended to $U_{1}$. Therefore, based on (10) and (11), the maximal transmission power factor of $\gamma_{2}$ is given by

$$
\gamma_{2}=\min \left\{\max \left\{0, \gamma_{21}\right\}, \max \left\{0, \gamma_{22}\right\}\right\},
$$

where

$$
\gamma_{21}=\frac{\rho^{2}\left|g_{n}\right|^{2}\left|h_{n 1}\right|^{2}-\varepsilon_{1} \rho\left|g_{n}\right|^{2}-\varepsilon_{1} \rho\left|h_{n 1}\right|^{2}-\varepsilon_{1}}{\rho^{2}\left|g_{n}\right|^{2}\left|h_{n 1}\right|^{2}\left(1+\varepsilon_{1}\right)},
$$

and

$$
\gamma_{22}=\frac{\rho^{2}\left|g_{n}\right|^{2}\left|h_{n 2}\right|^{2}-\varepsilon_{1} \rho\left|g_{n}\right|^{2}-\varepsilon_{1} \rho\left|h_{n 2}\right|^{2}-\varepsilon_{1}}{\rho^{2}\left|g_{n}\right|^{2}\left|h_{n 2}\right|^{2}\left(1+\varepsilon_{1}\right)} .
$$

In order to ensure that both $U_{1}$ and $U_{2}$ can always correctly decode signal $x_{1}$, the conditions $\rho^{2}\left|g_{n}\right|^{2}\left|h_{n 1}\right|^{2}-\varepsilon_{1} \rho\left|g_{n}\right|^{2}-$ $\varepsilon_{1} \rho\left|h_{n 1}\right|^{2}-\varepsilon_{1}>0$ and $\rho^{2}\left|g_{n}\right|^{2}\left|h_{n 2}\right|^{2}-\varepsilon_{1} \rho\left|g_{n}\right|^{2}-\varepsilon_{1} \rho\left|h_{n 2}\right|^{2}-$ $\varepsilon_{1}>0$ should be satisfied at the same time. Therefore, we can get the following constraint conditions

$$
\left|g_{n}\right|^{2}>\frac{\varepsilon_{1}}{\rho},\left|h_{n 1}\right|^{2}>G_{1}\left(\left|g_{n}\right|^{2}\right),\left|h_{n 2}\right|^{2}>G_{1}\left(\left|g_{n}\right|^{2}\right)
$$

where $G_{1}\left(\left|g_{n}\right|^{2}\right)=\frac{\varepsilon_{1}\left(\rho\left|g_{n}\right|^{2}+1\right)}{\rho\left(\rho\left|g_{n}\right|^{2}-\varepsilon_{1}\right)}$.

Based on (26), the target rate requirement can be realized of $U_{1}$, and the SIC can be correctly carried out at $U_{2}$ to remove the message for $U_{1}$. Therefore, the two-stage AF relaying selection scheme is defined as follows:

$$
S_{n}=\left\{n:\left|g_{n}\right|^{2}>\frac{\varepsilon_{1}}{\rho}, \min \left\{\left|h_{n 1}\right|^{2},\left|h_{n 2}\right|^{2}\right\}>G_{1}\left(\left|g_{n}\right|^{2}\right)\right\} .
$$

Note that $\gamma_{2}$ in (23) can be rewritten by applying (27) as follows:

$$
\gamma_{2}=\min \left\{\gamma_{21}, \gamma_{22}\right\}
$$

where $\gamma_{21}$ and $\gamma_{22}$ are defined in (24) and (25), respectively. Compared to the two-stage DF relaying scheme, it is interesting to find that the power allocate factors in the two-stage AF relaying scheme are always positive. In Section $\mathrm{V}$, both the analytical results and computer simulations show that the outage probability for users in cooperative NOMA networks 
with two-stage AF relaying can achieve better performance than those with two-stage DF relaying.

According to (12) and (28), the instantaneous rate at $U_{2}$ can be expressed as follows:

$$
\begin{aligned}
R_{2}^{n}=\min & \left\{\frac{1}{2} \log _{2}\left(1+\frac{\gamma_{21} \rho^{2}\left|g_{n}\right|^{2}\left|h_{n 2}\right|^{2}}{\rho\left|g_{n}\right|^{2}+\rho\left|h_{n 2}\right|^{2}+1}\right),\right. \\
& \left.\frac{1}{2} \log _{2}\left(1+\frac{\gamma_{22} \rho^{2}\left|g_{n}\right|^{2}\left|h_{n 2}\right|^{2}}{\rho\left|g_{n}\right|^{2}+\rho\left|h_{n 2}\right|^{2}+1}\right)\right\} .
\end{aligned}
$$

One best relay is selected from (27) to achieve the maximal rate for $U_{2}$, i.e.,

$$
n^{*}=\arg \max \left\{R_{2}^{n}, n \in S_{n}\right\}
$$

The following theorem provides an exact expression for the outage probability achieved by the two-stage AF relay selection in cooperative NOMA, as well as its diversity gain.

Theorem 2: The outage probability for $U_{2}$ in cooperative NOMA with the proposed two-stage AF relaying scheme is given by

$$
\mathrm{P}_{2}=\sum_{k=0}^{M}\left(\begin{array}{c}
M \\
k
\end{array}\right)\left(1-\mathrm{P}_{s}\right)^{M-k}\left(\mathrm{P}_{s}-q_{1}-q_{2}\right)^{k},
$$

where $q_{1}=\int_{0}^{1} \frac{\varepsilon_{1} e^{-\frac{g_{1}(t)}{\rho}} \rho t^{2}}{\sqrt{\frac{g_{2}(t)}{\rho^{2}}}} \mathbf{K}_{1}\left(\sqrt{\frac{g_{2}(t)}{\rho^{2}}}\right) d t, q_{2}=$ $2 \int_{0}^{1} \frac{\varepsilon_{1}\left(a_{2}+1\right) e^{-\frac{g_{1}(t)}{\rho}}}{\rho^{2} t^{2}} \mathbf{K}_{0}\left(\sqrt{\frac{g_{2}(t)}{\rho^{2}}}\right) d t, g_{1}(t)=a_{2}+\varepsilon_{1}\left(\frac{1}{t}+\right.$ $\left.\frac{a_{1}}{\varepsilon_{1}}\right)(t+1), g_{2}(t)=4 \varepsilon_{1}\left(a_{2}+1\right)\left(\frac{1}{t}+\frac{a_{1}}{\varepsilon_{1}}\right)(t+1), \mathrm{P}_{s}=$ $e^{-\frac{3 \varepsilon_{1}}{\rho}} \sqrt{\frac{8 \varepsilon_{1}\left(1+\varepsilon_{1}\right)}{\rho^{2}}} \mathbf{K}_{1}\left(\sqrt{\frac{8 \varepsilon_{1}\left(1+\varepsilon_{1}\right)}{\rho^{2}}}\right)$. The diversity order of $U_{2}$ is $M$.

Proof: See Appendix B.

It can be seen from Theorem 2 that a diversity order of $M$ can be achieved by cooperative NOMA with the two-stage AF relaying scheme, which is the same as the traditional OMA AF relaying strategy. However, the advantage of this new scheme for cooperative NOMA is that it allows the two users to share the same spectrum resource, and user $U_{1}$ with low target data rate requirements can be always served.

\section{Numerical Results}

This section provides computer simulations to evaluate the correctness of the outage performance of cooperative NOMA with the two-stage DF/AF relaying scheme. Suppose that the channels between any two nodes are independent and identically distributed complex Gaussian distributed, i.e., $\mathcal{C N}(0,1)$.

Fig. 1 and Fig. 2 plot the outage probability for cooperative NOMA with two-stage DF relaying and AF relaying, respectively, where cooperative OMA is also shown as a cooperative scheme. Observing the Fig. 1, one can conclude that compared to cooperative NOMA with two-stage maxmin relaying, the proposed two-stage DF scheme can realize better outage performance. Furthermore, both Fig. 1 and Fig. 2 manifest that cooperative NOMA can remarkably enhance the outage performance compared to cooperative OMA although all of them achieve the same diversity gain. In addition, when

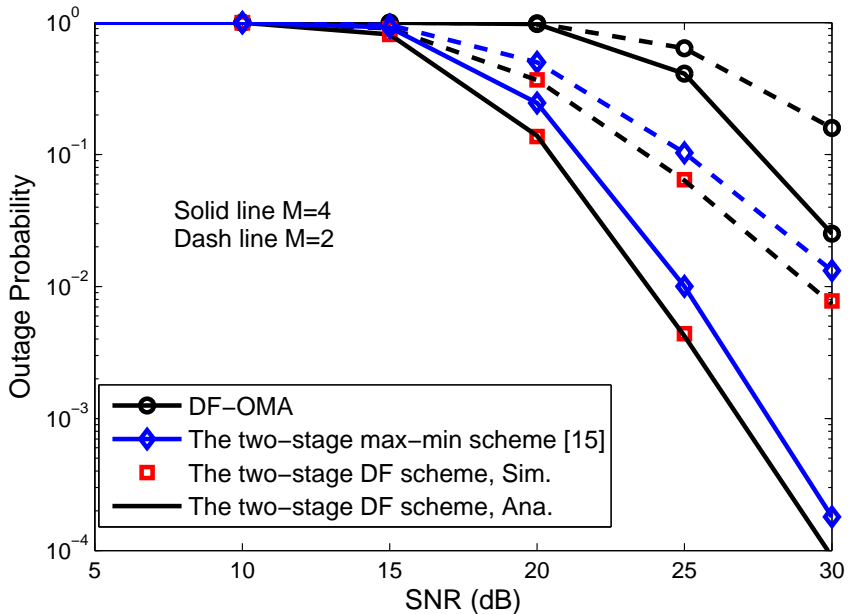

Fig. 1. Impact of $M$ on the outage performance for cooperative NOMA and OMA with different DF relaying schemes, where $R_{1}=$ $0.5 \mathrm{bits} / \mathrm{s} / \mathrm{Hz}, R_{2}=2 \mathrm{bits} / \mathrm{s} / \mathrm{Hz}$, the power allocation factors of two-stage max-min relay selection for cooperative NOMA are $\gamma_{1}=$ $\alpha_{1}=\frac{3}{4}$ [15], while the power allocation coifficient of two-stage DF relaying networks is $\gamma_{1}=\frac{3}{4}$ in Theorem 1 .

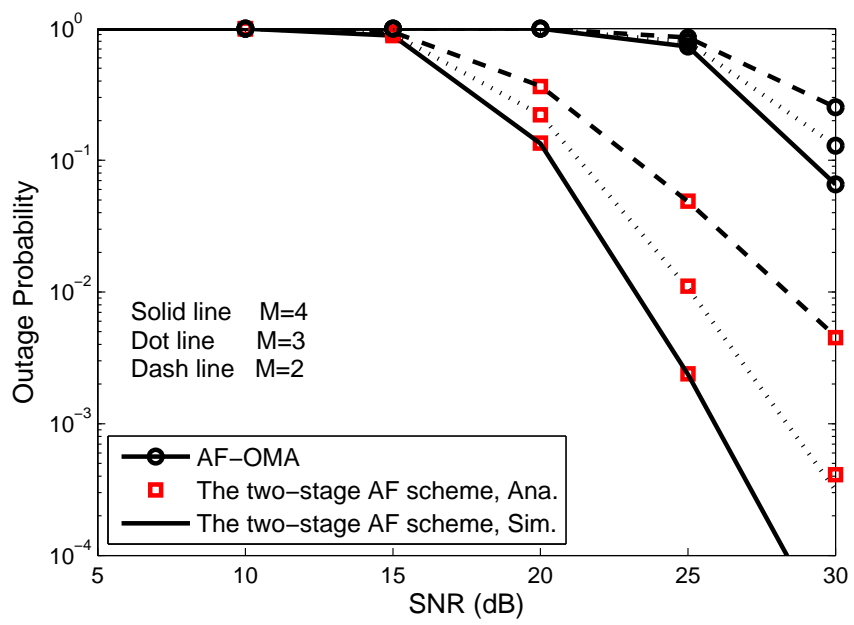

Fig. 2. Effect of $M$ on the outage performance for cooperative OMA and NOMA with AF relaying strategy, where $R_{1}=0.5 \mathrm{bits} / \mathrm{s} / \mathrm{Hz}$, $R_{2}=2$ bits $/ \mathrm{s} / \mathrm{Hz}$. The analytical results are based upon Theorem 2.

increasing the number of the relays $M$, the gap between the outage probabilities achieved by cooperative NOMA and OMA becomes large for both DF and AF relaying. In addition, it should be noticed that the analytical results in (21) and (31) are aligned with the Monte Carlo simulations in Fig. 1 and Fig. 2, respectively.

In Fig. 3, we investigate how the users' target data rates affect the outage probabilities of cooperative OMA with DF (AF) relaying and cooperative NOMA when two-stage DF (AF) relaying is used, respectively. It can be seen from Fig. 3 that in contrast to OMA with DF (AF) relaying, the outage probability for NOMA with two-stage DF (AF) relaying is significantly smaller, as increasing the gap between $R_{1}$ and $R_{2}$. The main reason for this phenomenon is that compared to OMA, the two users can be served at the same time in NOMA systems, which leads to an improvement of the 


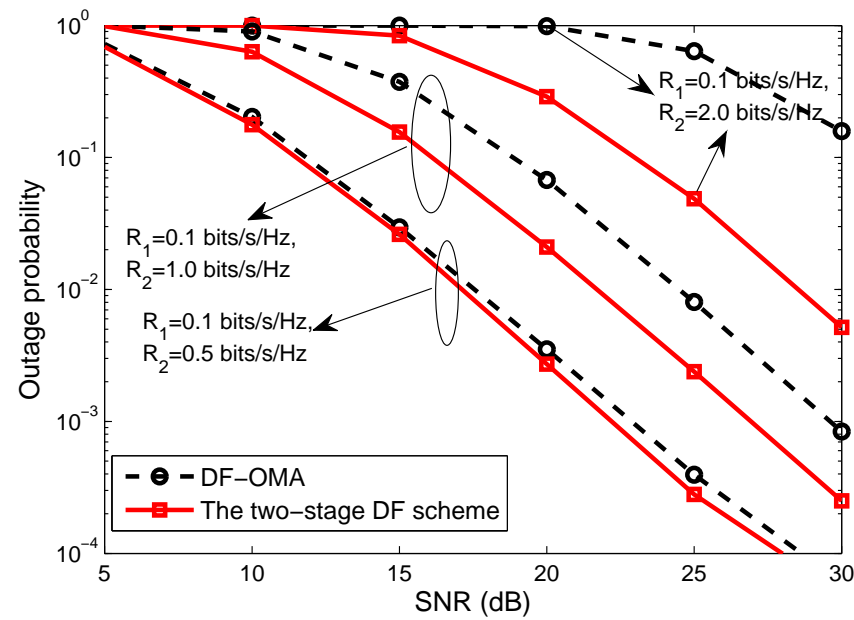

(a) two-stage DF relaying NOMA.

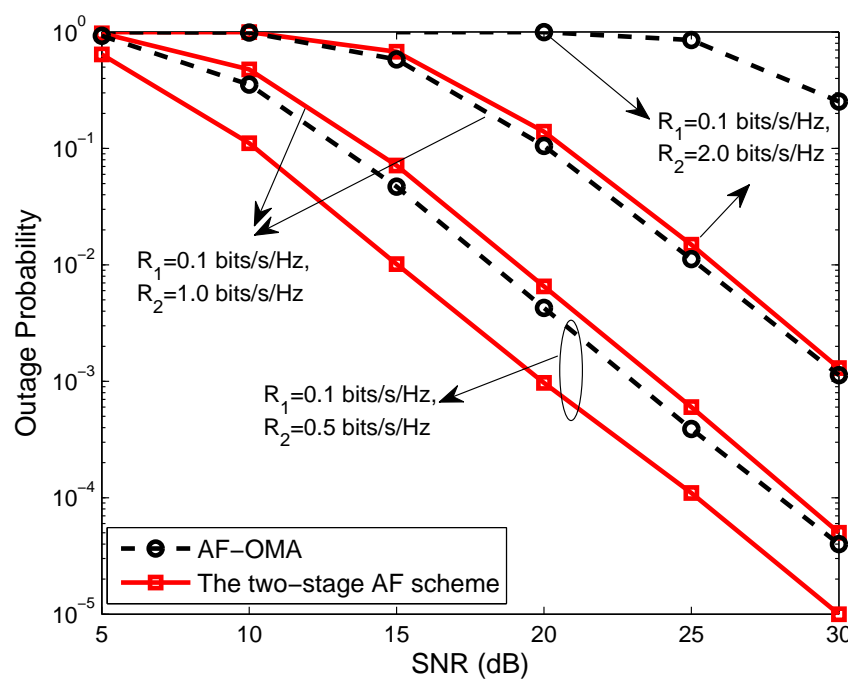

(b) two-stage AF relaying NOMA.

Fig. 3. Effect of users' target rates on cooperative two-stage AF/DF relaying NOMA with $M=2$.

spectral efficiency. Furthermore, the user $U_{2}$ in cooperative NOMA with two-stage relaying selection can achieve a higher date rate, since the SIC is likely to be successfully carried out at $U_{2}$ to remove the signal to $U_{1}$. Therefore, the two-stage relaying schemes for cooperative NOMA can be adapted to meet different $\mathrm{QoS}$ requirements.

In Fig. 4, we compare the outage performance for cooperative NOMA with two-stage AF/DF relaying to the two-stage max-min scheme by using different users' target rates and different numbers of relays. It can be observed from Fig. 4 that both two-stage AF and DF relaying schemes can achieve better outage performance than the two-stage max-min scheme [15] for different choices of $M$ and $R_{2}$. Furthermore, we can also see that the outage performance is enhanced significantly with low target data rates as $M$ increases. In addition, Fig. 4 also demonstrates that the two-stage AF relaying scheme can achieve the best outage performance among the three different relay schemes.

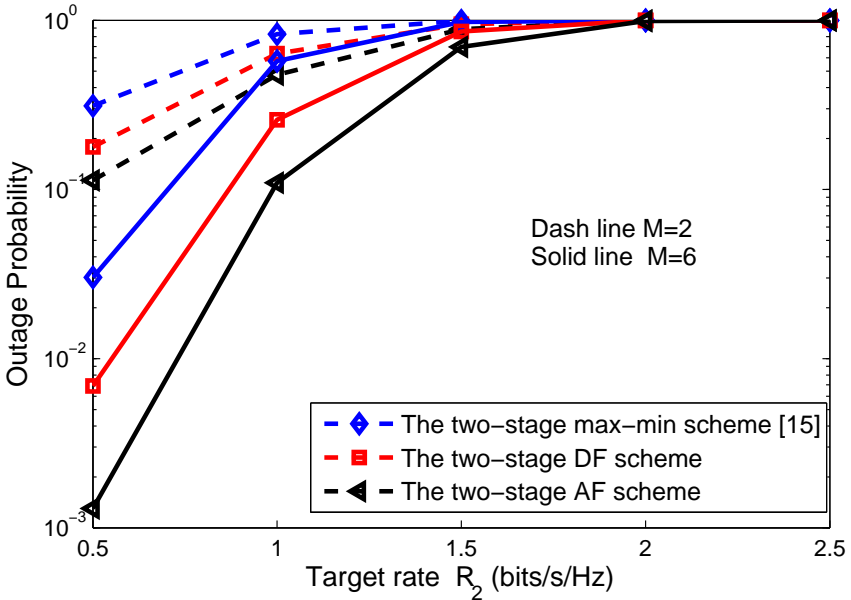

Fig. 4. Outage probability comparison between cooperative NOMA with $\mathrm{DF}$ and $\mathrm{AF}$ relaying, where $R_{1}=0.1 \mathrm{bits} / \mathrm{s} / \mathrm{Hz}, S N R=$ $10 \mathrm{~dB}$, the power allocation coifficients of two-stage DF relaying is $\gamma_{1}=\frac{3}{4}$. The power allocation coifficients of two-stage max-min are $\gamma_{1}=\alpha_{1}=\frac{3}{4}[15]$.

\section{Vi. Conclusions}

In this paper, we have proposed two kinds of two-stage DF and AF relay selection schemes for cooperative NOMA. For both two-stage DF and AF relaying schemes, we have derived exact and asymptotic analytical expressions for outage probability. The proposed two-stage cooperative NOMA schemes not only achieve full diversity order for both DF and AF relaying, but also yield better outage performance than cooperative OMA. In addition, compared to two-stage maxmin relay selection [15], the proposed two-stage DF or AF relay schemes can further improve the outage probability for cooperative NOMA.

In this paper, the unicast traffic and two users are considered for cooperative NOMA networks, one can extend the cooperative NOMA networks to the case with multiple users and multicast-unicast transmission [18]. In this case, for both the two-stage DF and AF relaying schemes, the rates of the multicast users are restricted to be higher than the target rate of the multicast message, then the best relay is selected to serve the unicast user. On the other hand, the proposed twostage AF/DF relaying scheme is superior to the two-stage max-min scheme, but it cannot achieve the optimal outage performance in cooperative NOMA networks. For the twostage DF relaying scheme, if full CSI is available at the source and the relays, the maximal ratio combining (MRC) strategy can be applied at the selected relays to achieve the optimal performance [19]. However, the disadvantage of the MRC strategy is that the global CSI at the relays and destinations should be feed to the source and the relays, respectively, which will significantly increase the system overhead. We will consider this interesting relaying scheme in the future, but it is out the scope of this paper. 


\section{APPENDIX A}

PROOF OF THEOREM 1

Based on (7), (17), and (18), the coverage probability of $U_{2}$ can be obtained as follows:

$$
\begin{aligned}
\overline{\mathrm{P}}_{2} & =\sum_{k=1}^{M} \operatorname{Pr}\left\{\frac{1}{2} \log _{2}\left(1+\alpha_{2} \rho\left|h_{n^{*} 2}\right|^{2}\right) \geq R_{2},\left|S_{n}\right|=k\right\} \\
& =\sum_{k=1}^{M} \underbrace{\operatorname{Pr}\left\{\alpha_{2} \geq \frac{\varepsilon_{2}}{\rho\left|h_{n^{*} 2}\right|^{2}}\left|S_{n}\right|=k\right\}}_{Q_{1}} \operatorname{Pr}\left\{\left|S_{n}\right|=k\right\} .
\end{aligned}
$$

For a given $k$, the above probability $Q_{1}$ can be evaluated by applying (20) as follows:

$$
\begin{aligned}
& Q_{1}=\operatorname{Pr}\left\{\left|h_{n^{*} 2}\right|^{2}>\frac{\varepsilon_{1}}{\rho}, \frac{\left|h_{n^{*} 1}\right|^{2}-\frac{\varepsilon_{1}}{\rho}}{\left|h_{n^{*} 1}\right|^{2}\left(\varepsilon_{1}+1\right)} \geq \frac{\varepsilon_{2}}{\rho\left|h_{n^{*} 2}\right|^{2}},\right. \\
& \left.\frac{\left|h_{n^{*} 2}\right|^{2}-\frac{\varepsilon_{1}}{\rho}}{\left|h_{n^{*} 2}\right|^{2}\left(\varepsilon_{1}+1\right)} \geq \frac{\varepsilon_{2}}{\rho\left|h_{n^{*} 2}\right|^{2}} \mid n^{*} \in S_{n}\right\} \\
& =\operatorname{Pr}\left\{\left|h_{n^{*} 1}\right|^{2} \geq \frac{\varepsilon_{1}\left|h_{n^{*} 2}\right|^{2}}{\rho\left|h_{n^{*} 2}\right|^{2}-a_{1}},\right. \\
& \left.\left|h_{n^{*} 2}\right|^{2}>\left.\frac{a_{2}}{\rho}|| h_{n^{*} 1}\right|^{2}>\frac{\varepsilon_{1}}{\rho}\right\} \\
& =\frac{\operatorname{Pr}\left\{\left|h_{n^{*} 1}\right|^{2} \geq \frac{\varepsilon_{1}\left|h_{n^{*} 2}\right|^{2}}{\rho\left|h_{n^{*} 2}\right|^{2}-a_{1}},\left|h_{n^{*} 2}\right|^{2}>\frac{a_{2}}{\rho}\right\}}{\operatorname{Pr}\left\{\left|h_{n^{*} 1}\right|^{2}>\frac{\varepsilon_{1}}{\rho}\right\}} \\
& =e^{\frac{\varepsilon_{1}}{\rho}} \int_{\frac{a_{2}}{\rho}}^{\infty} e^{-\frac{\varepsilon_{1} y}{\rho y-a_{1}}} f_{\left|h_{n^{*}}\right|^{2}}(y) d y \text {. }
\end{aligned}
$$

By using partial integration and the CDF of $\left|h_{n^{*} 2}\right|^{2}$, e.g. , $F_{\mid h_{\left.n^{*}\right|^{2}}}(y)=\left(1-e^{-y}\right)^{k}, Q_{1}$ in (33) can be further evaluated as follows:

$$
\begin{aligned}
Q_{1}=\left.e^{\frac{\varepsilon_{1}}{\rho}-\frac{\varepsilon_{1} y}{\rho y-a_{1}}} F_{\left|h_{\left.n^{*}\right|^{2}}\right|^{2}}(y)\right|_{\frac{a_{2}}{\rho}} ^{\infty} & \\
& -\varepsilon_{1} a_{1} e^{\frac{\varepsilon_{1}}{\rho}} \int_{\frac{a_{2}}{\rho}}^{\infty} \frac{e^{-\frac{\varepsilon_{1} y}{\rho y-a_{1}}}}{\left(\rho y-a_{1}\right)^{2}} F_{\left|h_{n^{*} 2}\right|^{2}}(y) d y .
\end{aligned}
$$

Let $t=\frac{\varepsilon_{1}}{\rho y-a_{1}}$, and the above $Q_{1}$ can be expressed as follows:

$$
\begin{aligned}
Q_{1}=1- & e^{-\frac{a_{1}}{\rho}}\left(1-e^{-\frac{a_{2}}{\rho}}\right)^{k} \\
& -\frac{a_{1}}{\rho} \int_{0}^{1} e^{-\frac{a_{1} t}{\rho}}\left(1-e^{-\frac{1}{\rho}\left(\frac{\varepsilon_{1}}{t}+a_{1}\right)}\right)^{k} d t .
\end{aligned}
$$

The probability of the event that one random relay is selected as an active relays in $S_{n}$ is given by

$$
\operatorname{Pr}\left\{\left|g_{n}\right|^{2} \geq \frac{\eta}{\rho},\left|h_{n 1}\right|^{2} \geq \frac{\varepsilon_{1}}{\rho}\right\}=e^{-\frac{a}{\rho}} .
$$

Then, the probability for the event that there are $k$ active relays in $S_{n}$ can be evaluated as follows:

$$
\operatorname{Pr}\left\{\left|S_{n}\right|=k\right\}=\left(\begin{array}{c}
M \\
k
\end{array}\right) e^{-\frac{a}{\rho} k}\left(1-e^{-\frac{a}{\rho}}\right)^{M-k} .
$$

Substituting (35) and (37) into (32), and using the fact that the outage probability $\mathrm{P}_{2}=1-\overline{\mathrm{P}}_{2}$, the first part of the theorem is proved.
In order to study the diversity order of the outage probability for $U_{2}$, assume $\rho \rightarrow \infty$ in the following part.

When $x \rightarrow 0$, the exponential function can be approximated by applying Taylor expanded as follows:

$$
e^{-x} \approx 1-x \text {. }
$$

Based on (38), the first term in (21) can be approximated as follows:

$$
\begin{aligned}
& \sum_{k=1}^{M}\left(\begin{array}{c}
M \\
k
\end{array}\right) e^{-\frac{a}{\rho} k}\left(1-e^{-\frac{a}{\rho}}\right)^{M-k} e^{-\frac{a_{1}}{\rho}}\left(1-e^{-\frac{a_{2}}{\rho}}\right)^{k} \\
& \approx \sum_{k=1}^{M}\left(\begin{array}{c}
M \\
k
\end{array}\right) \frac{a^{M-k}}{\rho^{M-k}} \frac{a_{2}^{k}}{\rho^{k}}=\frac{1}{\rho^{M}} \sum_{k=1}^{M}\left(\begin{array}{c}
M \\
k
\end{array}\right) a^{M-k} a_{2}^{k} .
\end{aligned}
$$

By using Gauss-Chebyshev integral [20], we can obtain the following integral approximation

$$
\begin{aligned}
& \int_{0}^{1} e^{-\frac{a_{1} t}{\rho}}\left(1-e^{-\frac{1}{\rho}\left(\frac{\varepsilon_{1}}{t}+a_{1}\right)}\right)^{k} d t \\
& \approx \frac{\pi}{2 N} \sum_{i=1}^{N}\left|\sin \frac{2 i-1}{N}\right| e^{-\frac{a_{1} t_{i}}{\rho}}\left(1-e^{-\frac{1}{\rho}\left(\frac{\varepsilon_{1}}{t_{i}}+a_{1}\right)}\right)^{k},
\end{aligned}
$$

where $t_{i}=\frac{1}{2}\left(1+\cos \frac{2 i-1}{N}\right)$, and $N$ is the Gauss-Chebyshev integral approximated sum term.

Based on (38) and (40), the last term in (21) can be approximated as follows:

$$
\begin{aligned}
& \frac{a_{1}}{\rho} \sum_{k=1}^{M}\left(\begin{array}{c}
M \\
k
\end{array}\right) e^{-\frac{a k}{\rho}}\left(1-e^{-\frac{a}{\rho}}\right)^{M-k} \\
& \quad \times \int_{0}^{1} e^{-\frac{a_{1} t}{\rho}}\left(1-e^{-\frac{1}{\rho}\left(\frac{\varepsilon_{1}}{t}+a_{1}\right)}\right)^{k} d t \\
& \approx a_{1} \sum_{k=1}^{M}\left(\begin{array}{c}
M \\
k
\end{array}\right) \frac{a^{M-k}}{\rho^{M-k+1}} \frac{\pi}{2 N} \sum_{i=1}^{N}\left|\sin \frac{2 i-1}{N}\right|\left(\frac{\varepsilon_{1}}{t_{i}}+a_{1}\right)^{k} \\
& =\frac{\pi a^{M-k}}{2 N} \frac{a_{1}}{\rho^{M+1}} \sum_{k=1}^{M}\left(\begin{array}{c}
M \\
k
\end{array}\right) \sum_{i=1}^{N}\left|\sin \frac{2 i-1}{N}\right|\left(\frac{\varepsilon_{1}}{t_{i}}+a_{1}\right)^{k} .
\end{aligned}
$$

Substributing (39), and (41) into (21), the second part of the theorem is proved.

\section{APPENDIX B}

\section{PROOF OF THEOREM 2}

Based on (12), (27), and (30), the outage probability for $U_{2}$ can be expressed as follows:

$$
\begin{aligned}
\mathrm{P}_{2}= & \sum_{k=1}^{M} \operatorname{Pr}\left\{R_{2}^{n^{*}}<R_{2},\left|S_{n}\right|=k\right\}+\operatorname{Pr}\left\{\left|S_{n}\right|=0\right\} \\
= & \sum_{k=1}^{M} \operatorname{Pr}\left\{R_{2}^{n^{*}}<R_{2}|| S_{n} \mid=k\right\} \operatorname{Pr}\left\{\left|S_{n}\right|=k\right\} \\
& \quad+\operatorname{Pr}\left\{\left|S_{n}\right|=0\right\} .
\end{aligned}
$$

The term $\operatorname{Pr}\left\{\left|S_{n}\right|=0\right\}$ in (42) can be expressed as follows:

$$
\operatorname{Pr}\left\{\left|S_{n}\right|=0\right\}=\left(1-\mathrm{P}_{s}\right)^{M},
$$


where $\mathrm{P}_{s}$ can be calculated as follows:

$$
\begin{aligned}
\mathrm{P}_{s} & =\operatorname{Pr}\left\{\left|g_{n}\right|^{2}>\frac{\varepsilon_{1}}{\rho},\left|h_{n 1}\right|^{2}>G_{1}\left(\left|g_{n}\right|^{2}\right),\left|h_{n 2}\right|^{2}>G_{1}\left(\left|g_{n}\right|^{2}\right)\right\} \\
& =\int_{\frac{\varepsilon_{1}}{\rho}}^{\infty} e^{-\frac{2 \varepsilon_{1}(\rho z+1)}{\rho\left(\rho z-\varepsilon_{1}\right)}-z} d z \\
& =e^{-\frac{3 \varepsilon_{1}}{\rho}} \sqrt{\frac{8 \varepsilon_{1}\left(1+\varepsilon_{1}\right)}{\rho^{2}}} \mathbf{K}_{1}\left(\sqrt{\frac{8 \varepsilon_{1}\left(1+\varepsilon_{1}\right)}{\rho^{2}}}\right)
\end{aligned}
$$

where the lase equation follows from the fact that $\int_{0}^{\infty} \exp \left(-\frac{\beta}{4 x}-\gamma x\right) d x=\sqrt{\frac{\beta}{\gamma}} \mathbf{K}_{1}(\sqrt{\beta \gamma})$ in [21, eq. (3.324)].

For a relay $n$ which is selected randomly from $S_{n}$ in (27), the coverage probability of $R_{2}^{n}$ in (29) can be evaluated by applying (28) as follows:

$$
\begin{aligned}
& \operatorname{Pr}\left\{R_{2}^{n} \geq R_{2} \mid n \in S_{n}\right\} \\
& =\operatorname{Pr}\left\{\frac{\varepsilon_{2}\left(\rho\left|g_{n}\right|^{2}+\rho\left|h_{n 2}\right|^{2}+1\right)}{\rho^{2}\left|g_{n}\right|^{2}\left|h_{n 2}\right|^{2}} \leq \min \left\{\gamma_{21}, \gamma_{22}\right\} \mid n \in S_{n}\right\} \\
& =\operatorname{Pr}\left\{\left|h_{n 2}\right|^{2}>G_{2}\left(\left|g_{n}\right|^{2}\right),\left|h_{n 1}\right|^{2}>G_{3}\left(\left|g_{n}\right|^{2},\left|h_{n 2}\right|^{2}\right),\right. \\
& \qquad\left|g_{n}\right|^{2}>\left.\frac{a_{2}}{\rho}|| g_{n}\right|^{2}>\frac{\varepsilon_{1}}{\rho},\left|h_{n 1}\right|^{2}>G_{1}\left(\left|g_{n}\right|^{2}\right), \\
& =\frac{1}{\mathrm{P}_{s}} \operatorname{Pr}\left\{\left|h_{n 2}\right|^{2}>G_{2}\left(\left|g_{n}\right|^{2}\right),\left|h_{n 1}\right|^{2}>G_{3}\left(\left|g_{n}\right|^{2},\left|h_{n 2}\right|^{2}\right),\right. \\
& =\frac{1}{\mathrm{P}_{s}} \int_{\frac{a_{2}}{\rho}}^{\infty} \int_{\frac{a_{2}(\rho z+1)}{\rho\left(\rho z-a_{2}\right)}}^{\infty} e^{-\frac{\varepsilon_{1} y(\rho z+1)}{\rho^{2} z y-a_{1} \rho z-a_{2} \rho y-a_{1}}-y} d y e^{-z} d z, \quad(45)
\end{aligned}
$$

where $\mathrm{P}_{s}$ is defined in (44), $G_{2}\left(\left|g_{n}\right|^{2}\right)=\frac{a_{2}\left(\rho\left|g_{n}\right|^{2}+1\right)}{\rho\left(\rho\left|g_{n}\right|^{2}-a_{2}\right)}$, $G_{3}\left(\left|g_{n}\right|^{2},\left|h_{n 2}\right|^{2}\right)=\frac{\varepsilon_{1}\left|h_{n 2}\right|^{2}\left(\rho\left|g_{n}\right|^{2}+1\right)}{\rho\left|h_{n 2}\right|^{2}\left(\rho\left|g_{n}\right|^{2}-a_{2}\right)-a_{1}\left(\rho\left|g_{n}\right|^{2}+1\right)}$.

Let $s=\rho z-a_{2}$ and $t=\frac{\varepsilon_{1}(\rho z+1)}{\rho y\left(\rho z-a_{2}\right)-a_{1}(\rho z+1)}$, the double integral in (45) can be further evaluated as follows:

$$
\begin{aligned}
& \int_{\frac{a_{2}}{\rho}}^{\infty} \int_{\frac{a_{2}\left(\rho z+a_{2}\right)}{\rho\left(\rho z-a_{2}\right)}}^{\infty} e^{-\frac{\varepsilon_{1} y(\rho z+1)}{\rho^{2} z y-a_{1} \rho z-a_{2} \rho y-a_{1}}-y} d y e^{-z} d z \\
& =\int_{0}^{\infty} \int_{0}^{1} \frac{\varepsilon_{1}\left(s+a_{2}+1\right)}{\rho^{2} s t^{2}} e^{-(1+t)\left(\frac{1}{t}+\frac{a_{1}}{\varepsilon_{1}}\right) \frac{\varepsilon_{1}\left(s+a_{2}+1\right)}{\rho s}} d t \\
& \quad \times e^{-\frac{s+a_{2}}{\rho} d s} \\
& =\underbrace{\int_{0}^{1} \frac{\varepsilon_{1} e^{-\frac{g_{1}(t)}{\rho}}}{\rho^{2} t^{2}} \int_{0}^{\infty} e^{-\frac{s}{\rho}-\frac{g_{2}(t)}{4 \rho s}} d s d t}_{q_{1}} \\
& \quad+\underbrace{\int_{0}^{1} \frac{\varepsilon_{1}\left(a_{2}+1\right) e^{-\frac{g_{1}(t)}{\rho}}}{\rho^{2} t^{2}} \int_{0}^{\infty} \frac{e^{-\frac{s}{\rho}-\frac{g_{2}(t)}{4 \rho s}}}{s} d s d t}_{q_{2}}
\end{aligned}
$$

where $g_{1}(t)=a_{2}+\varepsilon_{1}\left(\frac{1}{t}+\frac{a_{1}}{\varepsilon_{1}}\right)(t+1), g_{2}(t)=4 \varepsilon_{1}\left(a_{2}+1\right)\left(\frac{1}{t}+\right.$ $\left.\frac{a_{1}}{\varepsilon_{1}}\right)(t+1)$.

$q_{1}$ in (46) can be calculated by applying $\int_{0}^{\infty} \exp \left(-\frac{\beta}{4 x}-\right.$ $\gamma x) d x=\sqrt{\frac{\beta}{\gamma}} \mathbf{K}_{1}(\sqrt{\beta \gamma})$ in [21, eq. (3.324)] as follows:

$$
q_{1}=\int_{0}^{1} \frac{\varepsilon_{1} e^{-\frac{g_{1}(t)}{\rho}}}{\rho t^{2}} \sqrt{\frac{g_{2}(t)}{\rho^{2}}} \mathbf{K}_{1}\left(\sqrt{\frac{g_{2}(t)}{\rho^{2}}}\right) d t .
$$

$q_{2}$ in (46) can be calculated by applying $\mathbf{K}_{v}(z)=$ $\frac{1}{2}\left(\frac{z}{2}\right)^{v} \int_{0}^{\infty} \frac{\exp \left(-\frac{z^{2}}{4 t}-t\right)}{t^{v+1}} d t$ in [21, eq. (8.432)] as follows:

$$
q_{2}=2 \int_{0}^{1} \frac{\varepsilon_{1}\left(a_{2}+1\right) e^{-\frac{g_{1}(t)}{\rho}}}{\rho^{2} t^{2}} \mathbf{K}_{0}\left(\sqrt{\frac{g_{2}(t)}{\rho^{2}}}\right) d t .
$$

Substituting (47) and (48) into (45), we have

$$
\operatorname{Pr}\left\{R_{2}^{n} \geq R_{2} \mid n \in S_{n}\right\}=\frac{q_{1}+q_{2}}{\mathrm{P}_{s}} .
$$

By relying on (49), the first term in (42) can be evaluated as follows:

$$
\begin{aligned}
& \sum_{k=1}^{M} \operatorname{Pr}\left\{R_{2}^{n^{*}}<R_{2}|| S_{n} \mid=k\right\} \operatorname{Pr}\left\{\left|S_{n}\right|=k\right\} \\
& =\sum_{k=1}^{M}\left(1-\operatorname{Pr}\left\{R_{2}^{n} \geq R_{2} \mid n \in S_{n}\right\}\right)^{k} \operatorname{Pr}\left\{\left|S_{n}\right|=k\right\} \\
& =\sum_{k=1}^{M}\left(\begin{array}{c}
M \\
k
\end{array}\right) \mathrm{P}_{s}^{k}\left(1-\mathrm{P}_{s}\right)^{M-k}\left(1-\frac{q_{1}+q_{2}}{\mathrm{P}_{s}}\right)^{k},
\end{aligned}
$$

where $\operatorname{Pr}\left\{\left|S_{n}\right|=k\right\}=\left(\begin{array}{c}M \\ k\end{array}\right) \mathrm{P}_{s}^{k}\left(1-\mathrm{P}_{s}\right)^{M-k}$.

Substituting (43) and (50) into (42), and after some algebraic manipulations, we can obtain (31). The proof of the first part of the theorem is completed.

When $z \rightarrow 0$, the Bessel function $z \mathrm{~K}_{1}(z)$ can be approximated by using series representation as follows [21, eq. (8.446)]:

$$
\begin{aligned}
z \mathrm{~K}_{1}(z)= & 1+\sum_{k=0}^{\infty} \frac{\left(\frac{z}{2}\right)^{2 k+1} z}{k ! \Gamma(k+2)}\left(\ln \frac{z}{2}+\mathbf{C}\right) \\
& -\frac{1}{2} \sum_{l=0}^{\infty} \frac{\left(\frac{z}{2}\right)^{2 l+1} z}{l !(n+l) !}\left(\sum_{k=1}^{l} \frac{1}{k}+\sum_{k=1}^{l+1} \frac{1}{k}\right) \\
\approx & 1+\frac{z^{2}}{2} \ln \frac{z}{2} .
\end{aligned}
$$

Based on (51) and $\rho \rightarrow \infty, \mathrm{P}_{s}$ in (44) can be approximated as follows:

$$
\begin{aligned}
\mathrm{P}_{s} & =e^{-\frac{3 \varepsilon_{1}}{\rho}} \sqrt{\frac{8 \varepsilon_{1}\left(1+\varepsilon_{1}\right)}{\rho^{2}}} \mathbf{K}_{1}\left(\sqrt{\frac{8 \varepsilon_{1}\left(1+\varepsilon_{1}\right)}{\rho^{2}}}\right) \\
& \approx\left(1-\frac{3 \varepsilon_{1}}{\rho}\right)\left(1+\frac{2 \varepsilon_{1}\left(1+\varepsilon_{1}\right)}{\rho^{2}} \ln \frac{2 \varepsilon_{1}\left(1+\varepsilon_{1}\right)}{\rho^{2}}\right) \\
& \approx 1-\frac{3 \varepsilon_{1}}{\rho} .
\end{aligned}
$$

Based on (51), $q_{1}$ can be approximated as follows:

$$
\begin{aligned}
q_{1} & =\int_{0}^{1} \frac{\varepsilon_{1} e^{-\frac{g_{1}(t)}{\rho}}}{\rho t^{2}} \sqrt{\frac{g_{2}(t)}{\rho^{2}}} \mathbf{K}_{1}\left(\sqrt{\frac{g_{2}(t)}{\rho^{2}}}\right) d t \\
& \approx \frac{\varepsilon_{1} e^{-\frac{2 a_{2}}{\rho}}}{\rho} \int_{0}^{1} \frac{e^{-\frac{\varepsilon_{1}}{\rho t}-\frac{a_{1} t}{\rho}}}{t^{2}} d t .
\end{aligned}
$$


Let $v=\frac{1}{t}$, the above integral can be further evaluated as follows:

$$
\begin{aligned}
& \int_{0}^{1} \frac{e^{-\frac{\varepsilon_{1}}{\rho t}-\frac{a_{1} t}{\rho}}}{t^{2}} d t \\
& =\int_{0}^{\infty} e^{-\frac{\varepsilon_{1} v}{\rho}-\frac{a_{1}}{\rho v}} d v-\int_{0}^{1} e^{-\frac{\varepsilon_{1} v}{\rho}-\frac{a_{1}}{\rho v}} d v \\
& =\frac{\rho}{\varepsilon_{1}} \sqrt{\frac{4 a_{1} \varepsilon_{1}}{\rho^{2}}} \mathbf{K}_{1}\left(\sqrt{\frac{4 a_{1} \varepsilon_{1}}{\rho^{2}}}\right)-\int_{0}^{1} e^{-\frac{\varepsilon_{1} v}{\rho}-\frac{a_{1}}{\rho v}} d v .
\end{aligned}
$$

The Gauss Chebyshev technology [20] can be applied to approximate the above integral as follows:

$$
\begin{aligned}
\int_{0}^{1} e^{-\frac{\varepsilon_{1} v}{\rho}-\frac{a_{1}}{\rho v}} d v & \approx \frac{\pi}{2 N} \sum_{i=1}^{N}\left|\sin \frac{2 i-1}{N}\right| e^{-\frac{\varepsilon_{1} v}{\rho}-\frac{a_{1}}{\rho v}} \\
& \approx 1-\frac{\pi}{2 N} \sum_{i=1}^{N}\left|\sin \frac{2 i-1}{N}\right|\left(\varepsilon_{1} v_{i}+\frac{a_{1}}{v_{i}}\right) \frac{1}{\rho} .
\end{aligned}
$$

Substituting (54) and (55) into (53), $q_{1}$ can be approximated as follows:

$$
q_{1} \approx 1-\frac{2 a_{2}+\varepsilon_{1}}{\rho} .
$$

The series representation of Bessel function $\mathbf{K}_{0}(z)$ can be approximated with $z \rightarrow 0$ as follows [21, eq. (8.447)]:

$$
\begin{aligned}
\mathbf{K}_{0}(z) & =-\ln \left(\frac{z}{2}\right) \sum_{k=0}^{\infty} \frac{\left(\frac{z}{2}\right)^{2 k}}{(k !)^{2}}+\sum_{k=0}^{\infty} \frac{z^{2 k} \Psi(k+1)}{2^{2 k}(k !)^{2}} \\
& \approx-\ln \frac{z}{2}=\ln \frac{2}{z} .
\end{aligned}
$$

To approximate $q_{2}$, based on (57), we can formulate

$$
\begin{aligned}
q_{2} & =2 \int_{0}^{1} \frac{\varepsilon_{1}\left(a_{2}+1\right) e^{-\frac{g_{1}(t)}{\rho}}}{\rho^{2} t^{2}} \mathbf{K}_{0}\left(\sqrt{\frac{g_{2}(t)}{\rho^{2}}}\right) d t \\
& \approx \frac{\pi}{N} \sum_{i=1}^{N}\left|\sin \frac{2 i-1}{N}\right| \frac{\varepsilon_{1}\left(a_{2}+1\right) e^{-\frac{g_{1}\left(t_{i}\right)}{\rho}}}{\rho^{2} t_{i}^{2}} \mathbf{K}_{0}\left(\sqrt{\frac{g_{2}\left(t_{i}\right)}{\rho^{2}}}\right) \\
& \approx \frac{\pi}{2 N} \sum_{i=1}^{N}\left|\sin \frac{2 i-1}{N}\right| \frac{\varepsilon_{1}\left(a_{2}+1\right)}{\rho^{2} t_{i}^{2}} \ln \frac{4 \rho^{2}}{g_{2}\left(t_{i}\right)} .
\end{aligned}
$$

Based on (52), (56) and (58), we can find that

$$
\mathrm{P}_{s}-q_{1}-q_{2} \approx \frac{2 a_{1}}{\rho} .
$$

Substituting (52) and (59) into (31), the second part of theorem is proved.

\section{REFERENCES}

[1] "Proposed solutions for new radio access," Mobile and wireless communications enablers for the 2020 information society (METIS), Deliverable D.2.4, Helsinki Aalto Univ., Malte Schellmann, Huawei ERC, Espoo, Finland, Feb. 2015.

[2] Z. Ding, X. Lei, G. K. Karagiannidis, R. Schober, J. Yuan, and V. K. Bhargava, "A survey on non-orthogonal multiple access for $5 \mathrm{G}$ networks: Research challenges and future trends." [Online]. Available: http://arxiv.org/abs/1706.05347

[3] 3rd Generation Partnership Project (3GPP), "Study on downlink multiuser superposition transmission for LTE, TSG RAN meeting 67," Tech. Rep. RP-150496, Mar. 2015.
[4] Y. Saito, A. Benjebbour, Y. Kishiyama, and T. Nakamura, "System level performance evaluation of downlink non-orthogonal multiple access (NOMA)," in Proc. IEEE Annual Symposium on Personal, Indoor and Mobile Radio Communications (PIMRC), London, UK, Sept. 2013.

[5] Z. Ding, Z. Yang, P. Fan, and H. V. Poor, "On the performance of non-orthogonal multiple access in $5 \mathrm{G}$ systems with randomly deployed users," IEEE Sig. Process. Lett., vol. 21, no. 12, pp. 1501-1505, Dec. 2014.

[6] Z. Yang, Z. Ding, P. Fan, and G. K. Karagiannidis, "On the performance of non-orthogonal multiple access systems with partial channel information," IEEE Trans. Commun., vol. 64, no. 2, pp. 654-667, Feb. 2016.

[7] Z. Ding, M. Peng, and H. V. Poor, "Cooperative non-orthogonal multiple access in 5G systems," IEEE Commun. Lett., vol. 19, no. 8, pp. 14621465, Aug. 2015.

[8] J. Yang, P. Fan, T. Q. Duong, and X. Lei, "Exact performance of two-way af relaying in Nakagami-m fading environment," IEEE Trans. Wireless Commun., vol. 10, no. 3, pp. 980-987, Mar. 2011.

[9] C. Zhong, T. Ratnarajah, and K. K. Wong, "Outage analysis of decodeand-forward cognitive dual-hop systems with the interference constraint in Nakagami-m fading channels," IEEE Trans. Veh. Tech., vol. 60, no. 6, pp. 2875-2879, July 2011.

[10] J. Men, J. Ge, and C. Zhang, "Performance analysis of non-orthogonal multiple access for relaying networks over Nakagami-m fading channels," IEEE Trans. Veh. Technol., vol. 66, no. 2, pp. 1200-1208, Feb. 2017.

[11] S. Lee, D. B. da Costa, and T. Q. Duong, "Outage probability of nonorthogonal multiple access schemes with partial relay selection," in Proc. IEEE Annual International Symposium on Personal, Indoor, and Mobile Radio Communications (PIMRC), Valencia, Spain, Sept. 2016.

[12] J. Men and J. Ge, "Non-orthogonal multiple access for multiple-antenna relaying networks," IEEE Commun. Lett., vol. 19, no. 10, pp. 1686-1689, Oct. 2015.

[13] J. B. Kim and I. H. Lee, "Non-orthogonal multiple access in coordinated direct and relay transmission," IEEE Commun. Lett., vol. 19, no. 11, pp. 2037-2040, Nov. 2015.

[14] Y. Liu, Z. Ding, M. Elkashlan, and H. V. Poor, "Cooperative nonorthogonal multiple access with simultaneous wireless information and power transfer," IEEE J. Sel. Areas Commun., vol. 34, no. 4, pp. 938953, April 2016.

[15] Z. Ding, H. Dai, and H. V. Poor, "Relay selection for cooperative NOMA," IEEE Wireless Commun. Lett., vol. 5, no. 4, pp. 416-419, Aug. 2016.

[16] Z. Ding, L. Dai, and H. V. Poor, "MIMO-NOMA design for small packet transmission in the internet of things," IEEE Access, vol. 4, pp. 1393$1405,2016$.

[17] D. N. C. Tse, P. Viswanath, and L. Zheng, "Diversity-multiplexing tradeoff in multiple-access channels," IEEE Trans. Inform. Theory, vol. 50, pp. 1859-1874, Sept. 2004.

[18] Z. Ding, Z. Zhao, M. Peng, and H. V. Poor, "On the spectral efficiency and security enhancements of NOMA assisted multicast-unicast streaming," IEEE Trans. Commun., vol. 65, no. 7, pp. 3151-3163, July 2017.

[19] C. Yuen, W. H. Chin, Y. L. Guan, W. Chen, and T. Tee, "Bi-directional multi-antenna relay communications with wireless network coding," in Proc. IEEE Vehicular Technology Conference, VTC Spring, Singapore, May 2008.

[20] E. Hildebrand, Introduction to Numerical Analysis. Dover, New York, USA, 1987.

[21] I. S. Gradshteyn and I. M. Ryzhik, Table of Integrals, Series and Products, 6th ed. New York: Academic Press, 2000. 


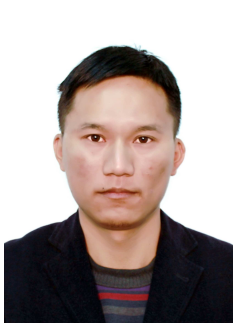

Zheng Yang (S'12) received the B.S. degree in mathematics from Minnan Normal University, Zhangzhou, in 2008, the M.S. degree in mathematics from Fujian Normal University, Fuzhou, China, in 2011, and the Ph.D. degree in information and communications engineering from Southwest Jiaotong University, Chengdu, China, in 2016.

Dr. Yang was a visiting Ph.D. student with the School of Electrical and Electronic Engineering, Newcastle University, Newcastle upon Tyne, U.K., in 2014. He is currently a Lecturer with the College of Photonic and Electronic Engineering, Fujian Normal University. His research interests include $5 \mathrm{G}$ networks, cooperative and energy harvesting networks, and signal design and coding.

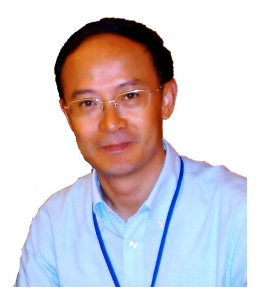

Pingzhi Fan (M'93-SM'99-F'15) received his $\mathrm{PhD}$ degree in Electronic Engineering from the Hull University, UK.

$\mathrm{He}$ is currently a professor and director of the institute of mobile communications, Southwest Jiaotong University, China. He is a recipient of the UK ORS Award, the Outstanding Young Scientist Award by NSFC, and the chief scientist of a national 973 research project. He served as general chair or TPC chair of a number of international conferences, and is the guest editor-in-chief, guest editor or editorial member of several international journals. He is the founding chair of IEEE VTS BJ Chapter and IEEE ComSoc CD Chapter, the founding chair of IEEE Chengdu Section. He also served as a board member of IEEE Region 10, IET(IEE) Council and IET Asia-Pacific Region. He has over 200 research papers published in various academic English journals (IEEE/IEE/IEICE, etc), and 8 books (incl. edited), and is the inventor of 22 granted patents.

His research interests include high mobility wireless communications, 5G technologies, wireless networks for big data, signal design and coding, etc. He is an IEEE VTS Distinguished Lecturer (2015-2017), and a fellow of IEEE, IET, CIE and CIC.

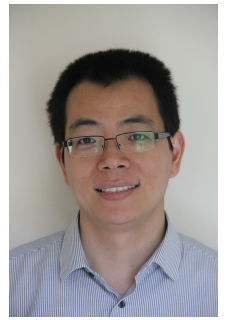

Zhiguo Ding (S'03-M'05-SM'15) received the B.Eng. degree in electrical engineering from the Beijing University of Posts and Telecommunications in 2000, and the Ph.D. degree in electrical engineering from Imperial College London in 2005. From 2005 to 2014, he was with Queens University Belfast, Imperial College London, and Newcastle University. From 2012 to 2016, he was an academic visitor with Princeton University. Since 2014, he has been a Chair Professor with Lancaster University.

His research interests are 5G networks, game theory, cooperative and energy harvesting networks, and statistical signal processing. He received the Best Paper Award at the IET Communication Conference on Wireless, Mobile and Computing in 2009, was an IEEE COMMUNICATIONS LETTERS Exemplary Reviewer in 2012, and held the EU Marie Curie Fellowship from 2012 to 2014. He is serving as an Editor for the IEEE TRANSACTIONS ON COMMUNICATIONS, the IEEE TRANSACTIONS ON VEHICULAR TECHNOLOGIES and the Journal of Wireless Communications and Mobile Computing. He was an Editor for the IEEE WIRELESS COMMUNICATION LETTERS and the IEEE COMMUNICATION LETTERS from 2013-2016. He is a Leading Guest Editor for the IEEE JOURNAL ON SELECTED AREAS IN COMMUNICATIONS Special Issue on Non-Orthogonal Multiple Access for 5G and a Guest Editor for the IEEE Wireless Communications Special Issue on Non-Orthogonal Multiple Access.

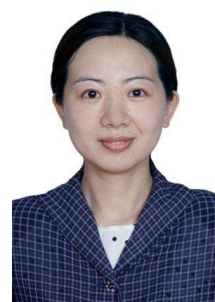

Yi Wu (M'07) received the B.Eng. degree in radio technology from Southeast University, Nanjing, China, in 1991; the M.S. degree in communications and information systems from Fuzhou University, Fuzhou, China, in 2004; and the Ph.D. degree in information and communication engineering from Southeast University, in 2013.

She is currently a Professor with the College of Photonic and Electronic Engineering, Fujian Normal University. Her research interests include $5 \mathrm{G}$ networks, vehicular ad hoc networks and communica- 